\title{
Large amplitude waves in a slug tracking scheme
}

\author{
A. De Leebeeck \& O. J. Nydal \\ Department of Energy and Process Engineering, \\ Norwegian University of Science and Technology (NTNU), Norway
}

\begin{abstract}
Large amplitude roll waves are incorporated into a previously developed slug tracking scheme for two phase gas-liquid pipe flow. The applicability of the tracking scheme to large amplitude waves is demonstrated with a simplified model for the waves. The waves are modelled analogous to slugs on a moving grid with corresponding wave velocities and a pressure variation determined using an orifice type relation. Slugs and waves in the tracking scheme are separated by regions of stratified flow, which are modelled on a stationary grid using the two-fluid model. The computational scheme is described, compared to experimental data on roll waves, and some wave dynamics such as waves developing to slugs and slugs decaying to waves are demonstrated.
\end{abstract}

Keywords: roll waves, tracking, two phase pipe flow, modelling.

\section{Introduction}

In two phase gas-liquid pipe flow, different flow regimes occur depending on gas and liquid phase velocities, fluid properties, and pipe geometries. Various numerical strategies exist for the different flow regimes in dynamic models. Slug flow, for example, can be treated with unit cell models (Bendiksen et al. [2]), in slug capturing (Bonizzi and Issa [3], Issa and Kempf [7], Renault [12]), or in tracking schemes [Taitel and Barnea [13], Nydal and Banerjee [11]). Although capturing schemes (Issa and Kempf [7], Bonizzi and Issa [3]) can model the initiation of slugs and roll waves, they require the use of fine grids which are computationally expensive and the large computational times are prohibitive for simulation in long pipelines. Tracking schemes, however, use 
orders of magnitude fewer grid points. Tracking schemes can also be suitable for plug simulations (Kjølaas [9]). A combination of capturing and tracking has also been tested by Renault [12].

Large roll waves can have similar scales and behavior to slug flow in that they transport liquid and have a propagating front. A simple model treating waves as moving objects in a similar way as for slugs is therefore desired in the tracking scheme. Similarities between slugs and roll waves such as a propagating front and a sloping tail have been observed experimentally in, for example, Johnson [8]. Pressure variations across wave fronts similar to slugs have also been measured (De Leebeeck et al. [4]). These experiments are used to develop a wave model including the observed pressure variation.

The slug tracking scheme of Kjølaas [9] is the starting point for incorporating wave tracking capabilities into a slug tracking scheme. Slug flow is modelled as alternating liquid slugs and bubbles with stratified flow. The two-fluid model is solved on a stationary staggered grid in bubbles, while integral momentum equations are solved in slugs on a moving grid. Before the addition of wave tracking, decaying slugs were replaced immediately with stratified flow. With the addition of wave tracking, slugs can decay into waves, modelled analogous to slugs with integral momentum equations and their own front and tail velocities.

The tracking scheme of $\mathrm{Hu}$ et al. [6] includes wave tracking with a liquid height profile behind waves and slugs, solving the two-fluid model in combination with modelling the wave front as a hydraulic jump (Hu et al. [6]). Other models for roll waves include, for example, Johnson [8] and Holmås [5] who solve the two-fluid model with modified friction terms in roll waves. Johnson [8] assumes a sequence of repeating "maximum amplitude" waves with a sharp front and includes a unique interfacial friction factor as part of a steady state solution. The model of Holmås [5] model is dynamic and includes increased turbulence at the wave front using a modified Biberg friction model.

In our scheme, we simplify waves and slugs as square objects that can be modelled dynamically on a coarse grid. The dynamics of the waves and slugs are determined from mass and momentum balances. The stratified gas regions between waves and slugs are solved with a two fluid model. A large grid gives square shaped bubbles. Slug and wave tails can be reproduced by refining the grid in the bubble region.

\section{Description of the model}

The wave tracking model builds on a slug tracking scheme (Kjølaas [9]) which is coded in $\mathrm{C}++$ using object oriented programming techniques. Slug flow is represented in one dimension with alternating slug objects that completely fill the pipe and stratified sections including both phases as shown in figure $1 \mathrm{~A}$. In stratified sections, the two-fluid model is solved on a stationary staggered grid where phase velocities are determined at section borders while pressure and masses are determined at section centers. Slug sections are modelled as moving 
objects where liquid phase velocity, slug length, front and tail velocities are determined from mass and momentum balances.

Waves, shown in figure 1B, are modelled in a similar way to slugs as moving objects and include a pressure variation due to liquid acceleration at the wave front. In the gas phase, the pressure variation across the wave front is modelled with an orifice type relation. Assuming that the pressure variation is the same in both phases, the phase velocities in the wave can be determined from the momentum balance equations. Front and tail velocities and liquid holdup are also determined in the wave assuming a fixed length as opposed to slugs which have a variable length.

A

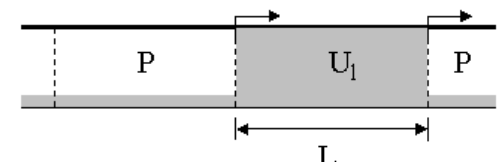

L

$\mathrm{B}$

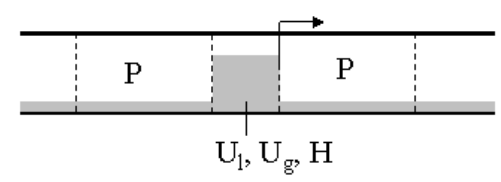

Figure 1: Schematic of models for A. slug flow and B. wave flow in the tracking scheme. The arrows indicate direction of flow. Dashed lines represent section borders. Gray - liquid phase. White - gas phase.

Gas flow in a slug is modelled using a slip relation, however, in a wave the gas phase flows through a gap between the liquid phase and the upper pipe wall. In this way, gas flow over a large wave can be thought of as similar to gas flow through an orifice, and therefore an orifice type relation is used in the gas momentum balance eqn (1) for waves. The orifice type relation, the second term in eqn (1), replaces the gas wall and interfacial friction terms. Eqn (1) is then used to determine the gas velocity in a wave by relating it to the pressure variation across the wave front.

$$
\begin{aligned}
& \frac{M_{g}}{\Delta t} \Delta U_{g} \\
& +(1-H) A \frac{1}{2} \frac{1}{C_{d}^{2}} \rho_{g}\left(1-\left(\frac{1-H}{1-H_{R}}\right)^{2}\right) \cdot\left(U_{g}^{n+1}-U_{\text {front }}\right)\left|U_{g}^{n}-U_{\text {front }}\right| \\
& =(1-H) A\left(P_{L}-P_{R}\right)-M_{g} g \sin \theta
\end{aligned}
$$

Using the same pressure variation across a wave front as in eqn (1), the liquid momentum balance eqn (2) is used to determine the liquid phase velocity in a wave. In the liquid phase, the main component giving pressure variation is the 
acceleration of the liquid at the wave front. There is also a contribution from liquid wall friction and gravity.

$$
\begin{aligned}
& \frac{M_{l}}{\Delta t} \Delta U_{l}+H \rho_{l} A\left(U_{l}^{n}-U_{\text {front }}\right)\left(U_{l R}^{n}-U_{l}^{n+1}\right) \\
& =H A\left(P_{L}-P_{R}\right)+\left(-\frac{1}{8} L S_{l} \lambda_{l} \rho_{l}\left|U_{l}^{n}\right| U_{l}^{n+1}\right)-M_{l} g \sin \theta
\end{aligned}
$$

Slugs are modelled as objects with moving boundaries, the front of a slug moves with a front velocity determined from a mass balance across the front while the tail moves with a bubble nose velocity. If the front velocity is greater than the tail velocity, the slug will grow in length, otherwise its length will decrease. Similarly, waves are modelled as moving objects but they have a fixed length of one to two pipe diameters and move with the wave front velocity. The front velocity of a wave is determined from the mass balance across the front, eqn (3), and given in eqn (4).

$$
\begin{aligned}
& H\left(U_{l}^{n+1}-U_{\text {front }}\right)=H_{R}\left(U_{l R}^{n}-U_{\text {front }}\right) \\
& U_{\text {front }}=\frac{H}{H-H_{R}} U_{l}^{n+1}-\frac{H_{R}}{H-H_{R}} U_{l R}^{n}
\end{aligned}
$$

One of the aims of the wave tracking scheme was to have a simplified model, therefore a simple wave tail speed relationship was desired. The wave tail speed is given in eqn (5).

$$
U_{\text {tail }}=1.2 U_{l}
$$

The factor of 1.2 allows for continuous transition between wave and slug flow. When the liquid holdup in a wave approaches unity, the liquid phase velocity in the wave approaches the mixture velocity. The bubble nose velocity or wave tail velocity is commonly related to the mixture velocity by a factor of 1.2 .

The mass balance equations in a wave or slug are the same, where the change in mass in a given time step is the difference in mass flux in and out. Eqns (6) and (7) are the liquid phase and gas phase mass balance equations respectively. The liquid holdup is given in eqn (8).

$$
\begin{gathered}
\frac{\Delta M_{l}}{\Delta t}=\left(M_{l}^{n}\left(U_{l}^{n+1}-U_{\text {tail }}\right) / L-M_{l}^{n}\left(U_{l}^{n+1}-U_{\text {front }}\right) / L\right) \\
\frac{\Delta M_{g}}{\Delta t}=\left(M_{g}^{n}\left(U_{g}^{n+1}-U_{\text {tail }}\right) / L-M_{g}^{n}\left(U_{g}^{n+1}-U_{\text {front }}\right) / L\right) \\
H=\frac{M_{l}^{n+1}}{A L \rho_{l}}
\end{gathered}
$$

Since wave fronts are modelled with a fixed length and they move at the wave front velocity, the wave tail velocity only appears in the mass balance eqns (6) and (7). If the front speed is larger than the tail speed the liquid mass in the wave will increase and vice versa. Therefore waves can grow or decay in amplitude. 


\subsection{Transitions and wave insertion}

Waves can be formed from stratified flow or they can form from decayed slugs. At the transition from stratified flow waves are inserted according to the inviscous Kelvin Helmholtz stability criteria, eqn (9). Neglecting surface tension and viscous effects, stratified flow is stable if (Barnea and Taitel [1], Lin and Hanratty [10]):

$$
\left(\frac{A_{l}}{\rho_{l} S_{i}}+\frac{A_{g}}{\rho_{g} S_{i}}\right)\left(\rho_{l}-\rho_{g}\right) g \cos \theta-\left(U_{g}-U_{l}\right)^{2}>0
$$

On the other hand, if a wave is dying, it will be removed when the holdup in the wave approaches the holdup in the stratified section in front of it.

A decaying slug will be converted to a wave when its length goes below a user defined minimum, i.e. one or two pipe diameters in length. In the reverse case where a wave grows to a slug, a wave will be converted to a slug if its holdup goes above a user defined maximum, e.g. a holdup of 0.99. Slugs can also form if two waves merge or a slug overtakes a slower moving wave.

\section{Results and discussion}

The roll wave tracking model has been compared with experiments that were conducted in the multiphase flow laboratory at NTNU in a $16 \mathrm{~m}$ long, $0.06 \mathrm{~m}$ I.D. pipe using air and water at atmospheric pressure (De Leebeeck et al. [4]). The experiments included pipe inclinations from -1 to 3 degrees, Usg from 2 to $11.5 \mathrm{~m} / \mathrm{s}$, and Usl from 0.04 to $0.52 \mathrm{~m} / \mathrm{s}$ where a mixture of waves and slugs occurred. Data for comparison include liquid holdup and pressure time traces, average wave velocities from cross correlation between holdup time traces, and pressure drop. One purpose of doing the experiments was to measure the pressure variation over a wave, as this is an assumption in the tracking model. This was confirmed in the experiments, and led to an estimate of the discharge coefficient in the orifice relation in eqn (1) of $C_{d}=0.2$ to 0.4 .

The simulations discussed here used a fixed grid size of 2 pipe diameters in wave fronts, a minimum of 20 and maximum of 100 pipe diameters in stratified sections. Pipe length, diameter, fluid properties, and a simulation time of $80 \mathrm{sec}$ were as in the experiments. A discharge coefficient giving the best approximation of experimental wave speed and pressure variation with value $C_{d}=0.2$ was used in the gas momentum equation for waves. The waves were inserted at a similar frequency to the experiments.

Figure 2 shows a plot of wave or slug velocity for all experiments and wave velocity from the model plotted against mixture velocity. The largest velocities are associated with slugs which have larger velocities than waves. The experiments with lower velocities contained more waves than slugs. Looking at the data qualitatively, the model gives wave velocities in the same range as the experiments. 
104 Computational Methods in Multiphase Flow V

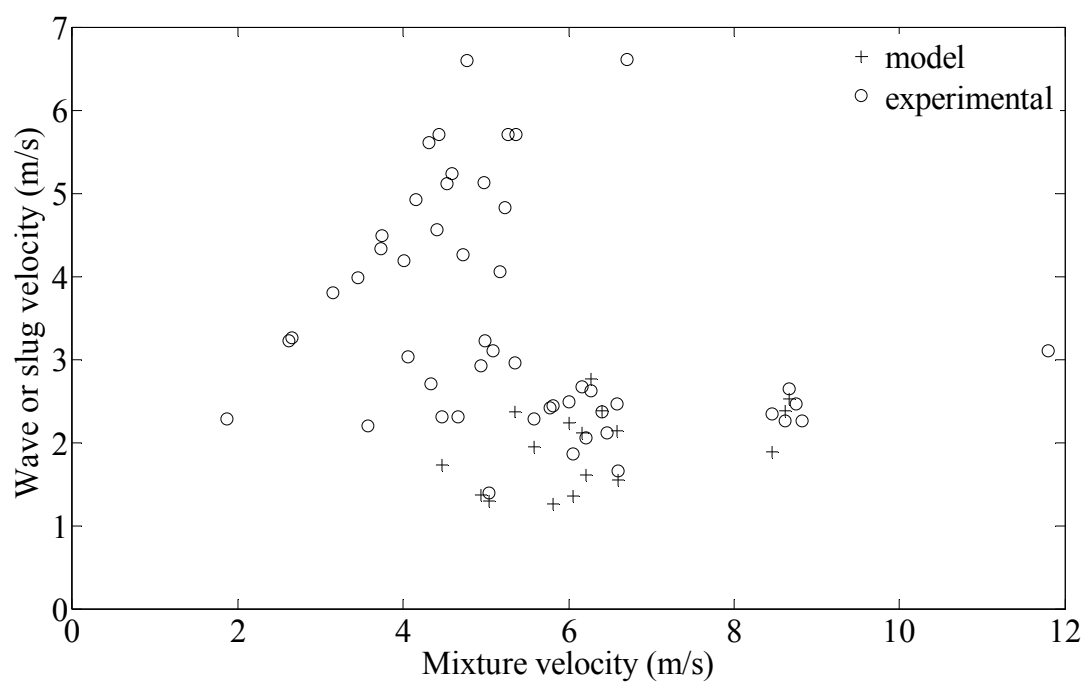

Figure 2: Experimental velocities compared with wave velocities from dynamic wave tracking simulations. For Umix $<4 \mathrm{~m} / \mathrm{s}$ : mainly slug flow. Umix $>4 \mathrm{~m} / \mathrm{s}$ : a mixture of waves and slugs.

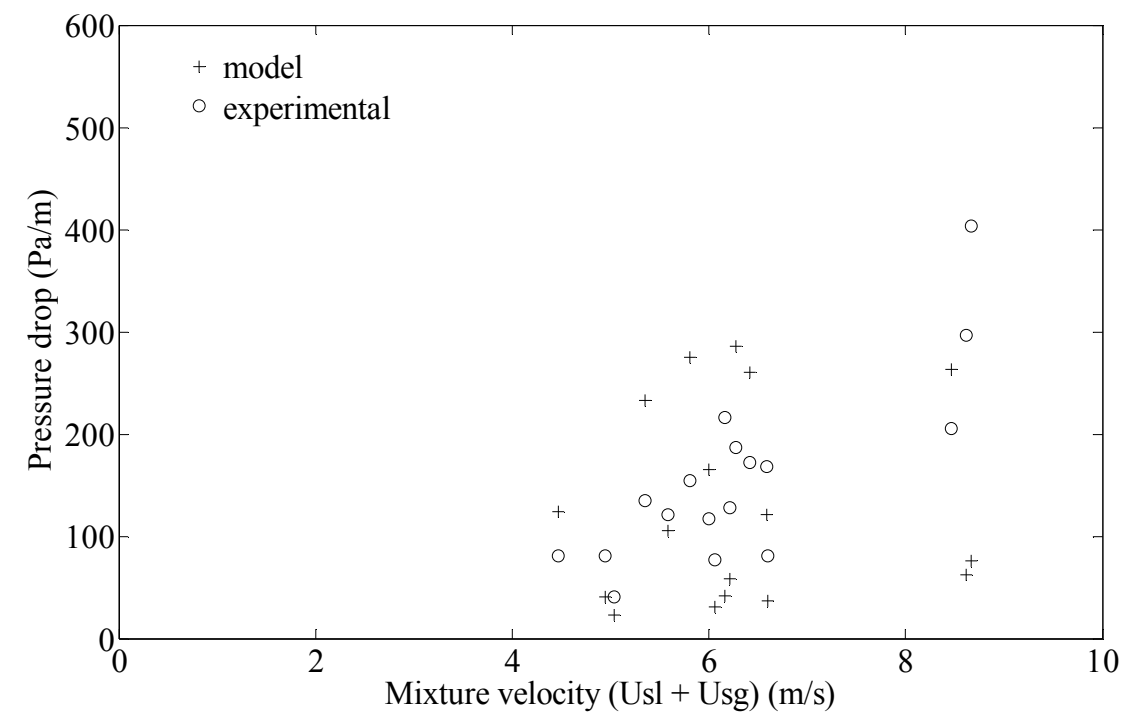

Figure 3: $\quad$ Experimental pressure drop compared with averaged pressure drop from tracking simulations.

In figure 3, the experimental and modelled pressure drops are compared. Although all of the simulations reproduced a pressure variation in waves, some of the pressure drops tended to be low compared to the experimental values. The 
magnitude of the pressure drop depends on the number of waves and slugs in the experiment or simulation. An experiment with more slugs will have a larger pressure drop than one with fewer. All the experiments contained a mixture of waves and slugs but some of the simulations, especially downward inclined, reproduced waves which did not grow to slugs resulting in low pressure drops.

One advantage of modelling waves in a tracking scheme is that coarse grids can be used allowing for longer pipe systems to be modelled. Using a coarse grid means that waves and slugs are modelled as square objects corresponding to the plots figure 4A, using a maximum stratified section length of 100 pipe diameters. Finer grids can be used, however, allowing for waves with more apparent tails, shown in figure $4 \mathrm{~B}$, as occurs in the experimental time traces, for example figure $4 \mathrm{C}$.
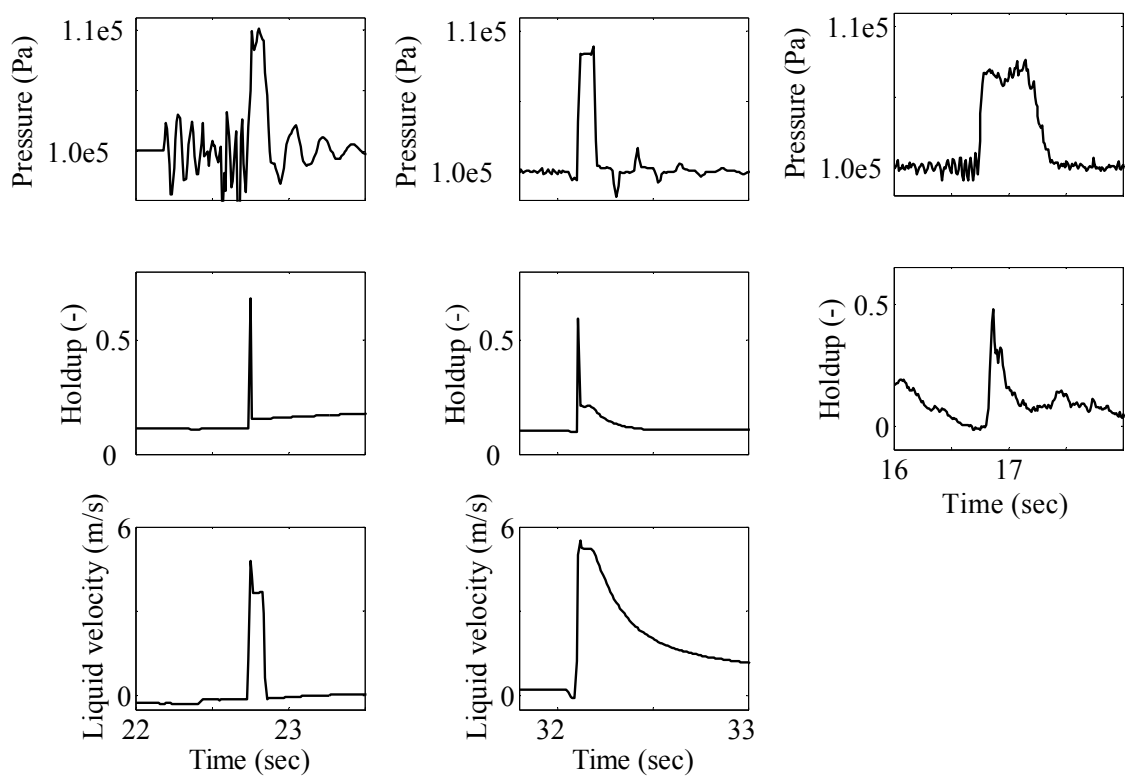

A

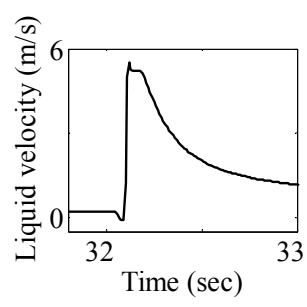

B

$\mathrm{C}$

Figure 4: Pressure, holdup and liquid velocity time traces where a wave passes at a given location in the pipe. A. A coarse grid with maximum length 100 pipe diameters. B. A fine grid with maximum length 10 pipe diameters. C. Experimental. Usg $=8.0$ $\mathrm{m} / \mathrm{s}, \mathrm{Usl}=0.1 \mathrm{~m} / \mathrm{s}, \theta=1$ degree.

Figure 5 shows how an experimental holdup time trace compares to a simulated time trace on a fine grid at the same Usg, Usl and pipe inclination. The model time trace in figure 5B shows a mixture of slugs and waves of various sizes occurring in the pipe as well as the shape of the waves.

Wave dynamics such as waves growing to slugs, or slugs decaying to waves is inherent in the tracking model. Examples of a wave growing to a slug and a 

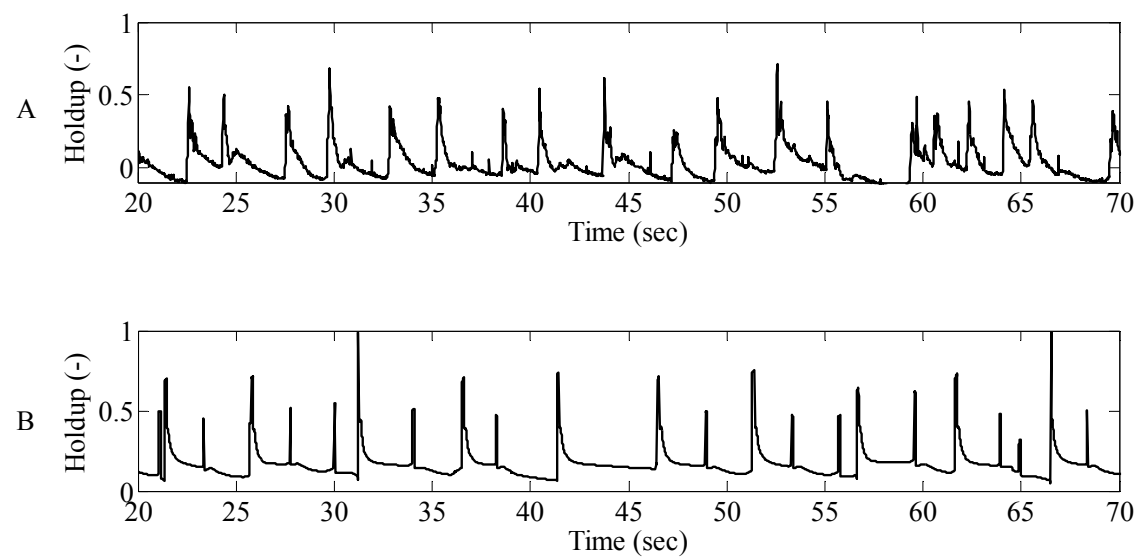

Figure 5: Holdup time traces A. experimental and B. simulation using a fine grid with maximum length 10 pipe diameters. Usg $=6.09 \mathrm{~m} / \mathrm{s}$, Usl $=0.18 \mathrm{~m} / \mathrm{s}, \theta=2$ degrees.

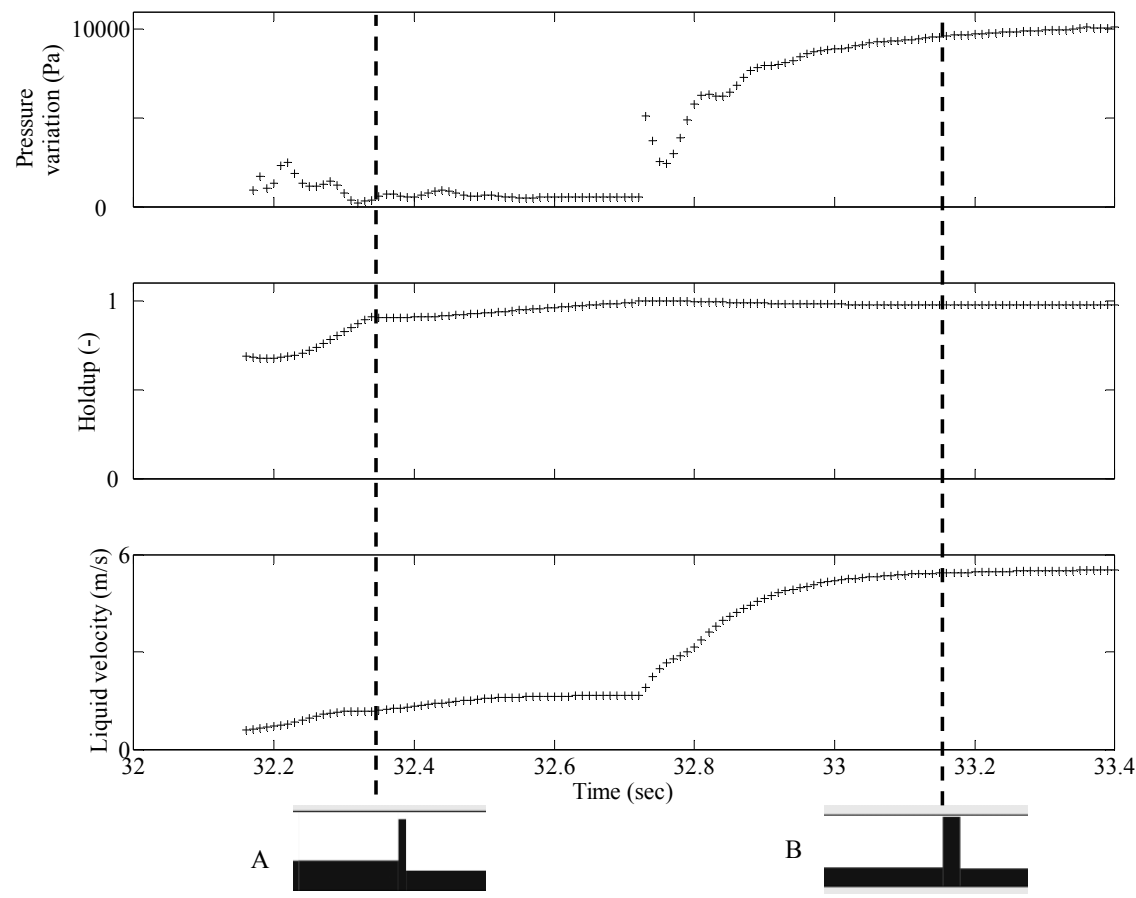

Figure 6: $\quad$ Pressure variation, liquid holdup and liquid velocity in a wave vs. time for A. a wave growing to B. a slug. Usg $=6.01 \mathrm{~m} / \mathrm{s}$, Usl $=$ $0.2 \mathrm{~m} / \mathrm{s}$, horizontal pipe. 

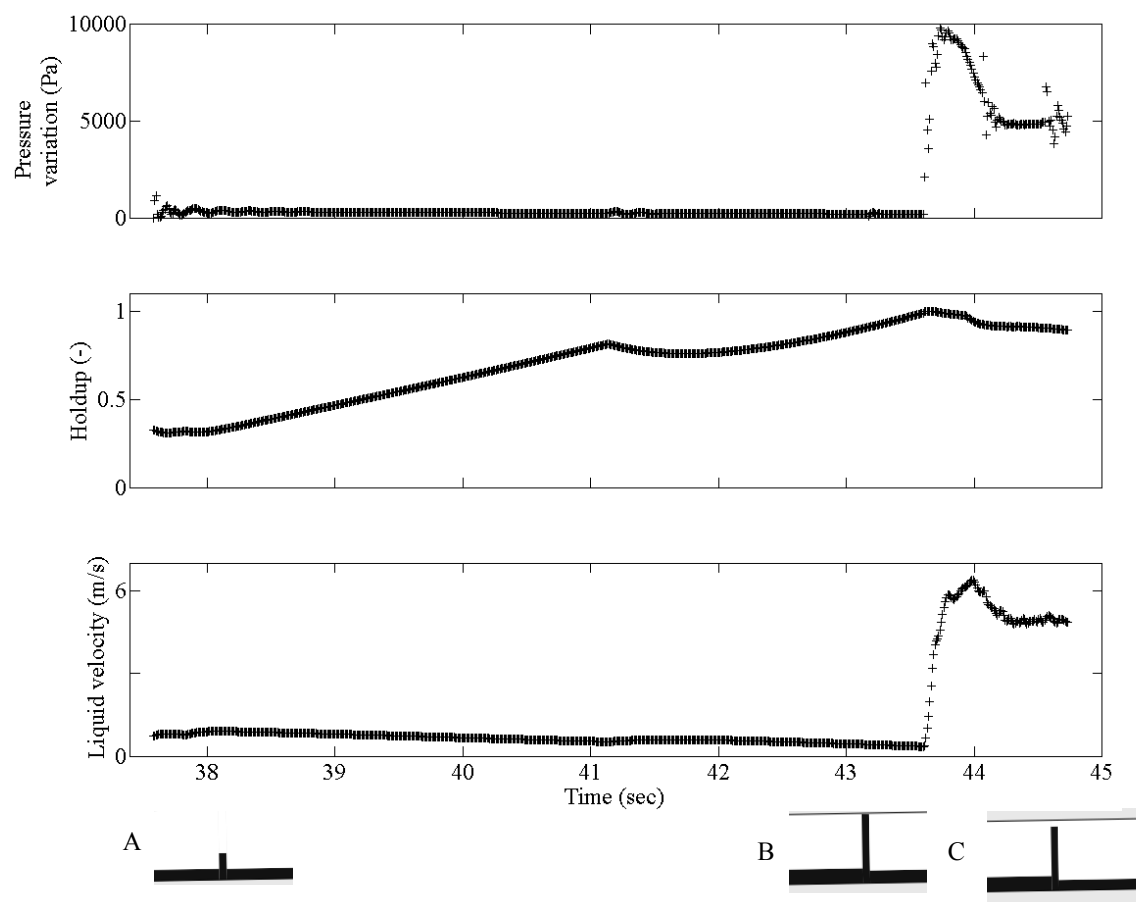

Figure 7: $\quad$ Pressure variation, liquid holdup, and liquid velocity in a wave vs. time for A. a wave growing to B. a slug and then decaying to C. a wave again. Usg $=5.87 \mathrm{~m} / \mathrm{s}$, Usl $=0.13 \mathrm{~m} / \mathrm{s}, \theta=1$ degrees.

wave which becomes a slug and then decays into a wave again are shown in figures 6 and 7 respectively. The pressure variation across the wave, liquid holdup and velocity in the wave object as it moves are plotted against time in both figures. When a wave becomes a slug the pressure variation across it and the liquid velocity increase, and the holdup approaches one. In figure 7, when the slug decays again, pressure variation, liquid velocity and holdup decrease. The time traces are cut off when the wave or slug exits the pipe.

\section{Conclusions}

A model for large roll waves has been implemented and tested in a slug tracking scheme. The model introduces an orifice type relation for pressure variation across the wave front and a simplified relationship for wave speed in a similar way as for slug flow. Computations have been demonstrated in comparison to experimental data on roll waves in two-phase air-water pipe flow at atmospheric pressure. The model gives a reasonable approximation of wave speed and pressure variations in waves. Looking at pressure drops, modelled pressure drops 
are sometimes low compared to experiments due to a difference in the number of waves and slugs in the pipe.

The tracking scheme can run with a coarse grid which allows simulation in longer pipes but means that waves and slugs are modelled as square objects without tails. A finer grid allows a more physical representation of waves with tails. The model includes wave dynamics such as a wave growing to a slug or a slug decaying to a wave.

\title{
5 List of Symbols
}

\author{
$A$ area, $\mathrm{m}^{2}$ \\ $C_{d}$ discharge coefficient \\ $g$ gravity, $9.81 \mathrm{~m} / \mathrm{s}^{2}$ \\ $H$ liquid holdup \\ I.D. internal diameter \\ $L \quad$ length of section, $\mathrm{m}$ \\ $M \quad$ mass, $\mathrm{kg}$ \\ $P$ pressure, $\mathrm{Pa}$ \\ $S \quad$ wetted perimeter, $\mathrm{m}$ \\ $t$ time, sec \\ $U$ velocity, $\mathrm{m} / \mathrm{s}$ \\ Umix mixture velocity, $\mathrm{m} / \mathrm{s}$ \\ Usg superficial gas velocity, $\mathrm{m} / \mathrm{s}$ \\ Usl superficial liquid velocity, $\mathrm{m} / \mathrm{s}$
}

Greek symbols
$\Delta \quad$ change in a given quantity
$\lambda$ friction factor
$\theta \quad$ angle of pipe inclination, degrees
$\rho$ density, $\mathrm{kg} / \mathrm{m}^{3}$

\section{Superscripts}

n current time step

$\mathrm{n}+1 \quad$ next time step

\section{Subscripts}

front front of a wave or slug

g gas phase

i interface

1 liquid phase

L left section

$\mathrm{R}$ right section

tail tail of a wave of slug 


\section{Acknowledgement}

Financial support from Total E\&P Norge is gratefully acknowledged by A. De Leebeeck.

\section{References}

[1] Barnea, D. \& Taitel, Y., Kelvin-Helmholtz stability criteria for stratified flow: Viscous versus non-viscous (inviscid) approaches, International Journal of Multiphase flow, 19, pp. 639-649, 1993.

[2] Bendiksen, K.H., Malnes, D. \& Nydal, O.J., On the modelling of slug flow, Chemical Engineering Communications, 141, pp. 71-102, 1996.

[3] Bonizzi, M. \& Issa, R.I., A model for simulating gas bubble entrainment in two-phase horizontal slug flow, International Journal of Multiphase flow, 29, pp. 1685-1717, 2003.

[4] De Leebeeck, A., Gaarder, A.H. \& Nydal, O.J., Experiments on Roll Waves in Air-Water Pipe Flow, $16^{\text {th }}$ Australasian Fluid Mechanics Conference, Gold Coast, Australia, 2007.

[5] Holmås, H., Numerical simulation of waves in two phase pipe flow using 1D two-fluid models, Doctoral dissertation, University of Oslo, 2008.

[6] Hu, B., Stewart, C., Manfield, P.D., Ujang, P.M., Hale, C.P., Lawrence, C.J. \& Hewitt, G.F., A Model for Tracking the Evolution of Slugs and Waves in Straight Pipelines, $6^{\text {th }}$ International Conference on Mulitphase Flow, Leipzig, Germany, 2007.

[7] Issa, R.I. \& Kempf, M.H.W., Simulation of slug flow in horizontal and nearly horizontal pipes with the two-fluid model, International Journal of Multiphase flow, 29, pp. 69-95, 2003.

[8] Johnson, G.W., A Study of Stratified Gas-Liquid Pipe Flow, Doctoral dissertation, University of Oslo, 2005.

[9] Kjølaas, J., Plug propagation in multiphase flow, Doctoral thesis, Norwegian University of Science and Technology, 2007.

[10] Lin, P.Y. \& Hanratty, T.J., Prediction of the initiation of slugs with linear stability theory, International Journal of Multiphase flow, 12, pp. 79-98, 1986.

[11] Nydal, O.J. \& Banerjee, S., Dynamic slug tracking simulation for gas-liquid flow in pipes, Chemical Engineering Communications, 141-142, pp. 13-39, 1996.

[12] Renault, F., A Lagrangian slug capturing scheme for gas-liquid flows in pipes, Doctoral thesis, Norwegian University of Science and Technology, 2007.

[13] Taitel, Y. \& Barnea D., Effect of gas compressibility on a slug tracking model, Chemical Engineering Science, 53(11), pp. 2089-2097, 1998. 